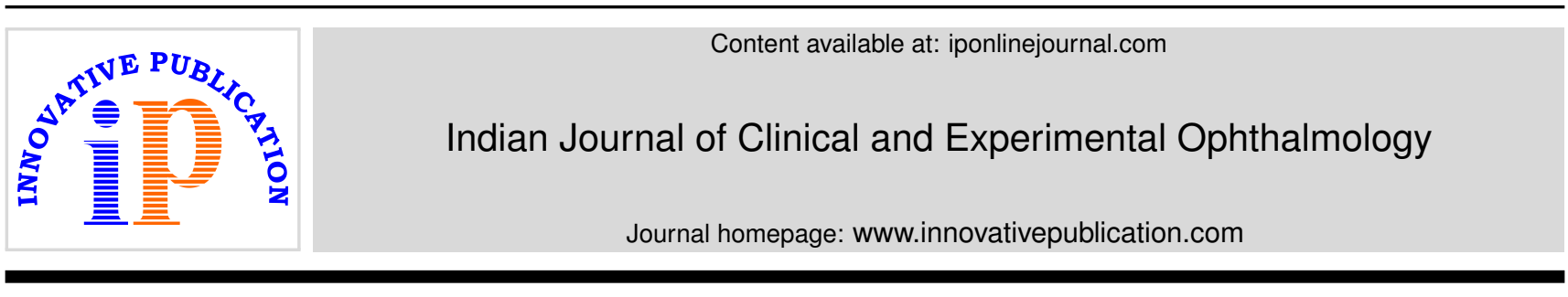

Original Research Article

\title{
Assessing the effect of anti-glaucoma drug on corneal morphology and tear film function
}

\author{
Veshal Madan $^{1, *}$, Shubhangi Katare ${ }^{1}$, Neeta Mishra ${ }^{1}$ \\ ${ }^{1}$ Dept. of Optometry and Visual Sciences, Dr. D. Y. Patil Institute of Optometry \& Visual Sciences, Pune, Maharashtra, India
}

\section{A R T I C L E I N F O}

\section{Article history:}

Received 28-01-2020

Accepted 09-05-2020

Available online 16-06-2020

\section{Keywords:}

Anti glaucoma drugs

Corneal morphology

Tear film

\begin{abstract}
A B S T R A C T
Purpose: To assess the effect of anti-glaucoma drug on corneal morphology and tear film function.

Materials and Methods: This is a prospective comparative study between two groups. 50 eyes of 25 patients using one or more topical anti glaucoma drugs for at least 6 months or more and Group 2- 50 eyes of 25 normal subjects as controls who were not having any ocular problems. Both groups were similar regarding age and gender characteristics. Demographic information were obtained from patient's medical records. Comprehensive ocular examination like Intraocular pressure (IOP), Tear breakup time (TBUT), Schirmer's II test (with anaesthesia), Central corneal thickness (CCT), Endothelial cell count (specular microscopy), Corneal staining were done for both the groups.

Results: All 50 patients (100eyes) included in the study and completed all the tests parameter involved in the study. Mann-Whitney U value for IOP is $(\mathrm{p}=0.001)$, CCT is $(\mathrm{p}=0.002)$, Endothelial cell count is $(\mathrm{p}=0.001)$, Schirmer's II is $(\mathrm{p}=0.001)$ and TBUT is $(\mathrm{p}=0.001)$, respectively and all this were statistically significant. For corneal staining Fisher's Exact Test value is $19.874(\mathrm{p}=0.001)$ is statistically significant. For CCT compared with the different diagnosis the Chi-Square value is $36.915(\mathrm{p}=0.001)$ is statistically significant. For Endothelial cell count compared with the different diagnosis the Chi-Square value is 11.876 $(\mathrm{p}=0.003)$ is statistically significant. CCT and Endothelial cell count was more in Ocular hypertension as compared to the other diagnosis.

Conclusion: Patients using anti-glaucoma drugs showed $64 \%$ mild to moderate and $20 \%$ severe dryness in the eyes. CCT and Endothelial cell count was more in Ocular hypertension as compared to the other diagnosis. Increased CCT may give an artificially high IOP and must be considered when developing a treatment approach for patients with ocular hypertension. Therefore, necessary to have complete evaluation of tear film and cornea while using the anti-glaucoma medication from long time. So that we can prescribe lubricating drops with the anti-glaucoma drugs.
\end{abstract}

(C) 2020 Published by Innovative Publication. This is an open access article under the CC BY-NC license (https://creativecommons.org/licenses/by-nc/4.0/)

\section{Introduction}

In today's world glaucoma is one of the leading causes of blindness with an estimated prevalence of $2.65 \%$ in the population over $40 .^{1}$ The majority of the glaucoma patients receive medical treatment in the form of topical eye drops. Glaucoma is a progressive disease which requires long term treatment sometimes often rest of life. Some of them require multiple medications. ${ }^{2}$ Anti-glaucoma medications are generally well tolerated. Preservatives are used in

\footnotetext{
* Corresponding author.

E-mail address: director.optom@dpu.edu.in (V. Madan).
}

these medications to maintain sterility. Long term use of these preserved anti-glaucoma medications has shown an adverse effect on conjunctiva and cornea, leading to an increase or worsen the risk of developing the ocular surface disease. ${ }^{3}$ Patients with glaucoma often remain on longterm therapy with varying numbers of pressure-lowering glaucoma medications that have been studied extensively and have been shown to cause molecular changes. From a molecular perspective, however, glaucoma medications do cause changes in corneal endothelial cells. ${ }^{4}$ Ocular adverse effects that have been noted for the use of timolol-included tear film alterations, hyperemia, burning 
sensation, corneal punctate erosions, and corneal anesthesia. Topical latanoprost causes cystoid macular edema, allergic contact dermatitis, hyperemia, hypertrichosis, and increased pigmentation of the iris. ${ }^{5}$ The prolonged and delayed effect of BAC is because of the incorporation and persistence of the molecules of BAC in cell membranes. ${ }^{6}$ This affects the tear film causing instability of the lipid layer ${ }^{7}$ Hence, BAC toxicity include direct damage to the cornea, a deterrent effect causing loss of tear film stability. ${ }^{6}$ It is, therefore, necessary to have a complete evaluation of tear film and cornea while using the anti-glaucoma medication for a long time. Present study uses latest non-invasive technique i.e., oculus pentacam to measure central corneal thickness and Schirmer's II test and endothelial cell count has been covered for the first time. Since limited data is available on the "Effect of anti-glaucoma drug on the cornea and tear film" the present study will provide more information on the Effect of anti-glaucoma drug on corneal morphology and tear film function.

\section{Materials and Methods}

This prospective comparative study was conducted at "PBMA's H.V. Desai eye hospital, Pune"between two groups of patients using one or more topical antiglaucoma drugs for at least 6 months or more in Group 1- 50 eyes of 25 patients and Group 2- 50 eyes of 25 normal subjects as controls who were not having any ocular problems. Patients recruited based on the pre-set inclusion and exclusion criteria.

For patients using antiglaucoma drugs

\subsection{Inclusion criteria}

1. Patients whose age is $\geq 20$ and $\leq 55$ years.

2. Patients with primary angle-closure glaucoma, ocular hypertension and primary open-angle glaucoma.

3. Patients using one or more topical anti-glaucoma drug as single or combination treatment with one or more antiglaucoma topical preservatives (timolol $0.5 \%$, brimonidine $0.1 \%$, latanoprost $0.005 \%$ ).

4. Patients using topical drugs for $\geq 6$ months.

\subsection{Exclusion criteria}

1. Patients who have undergone any Lasik or intraocular surgery.

2. Patients with any conditions known to affect the tear film.

3. Patients with any systemic conditions known to affect the tear film and cornea.

4. Patients with secondary glaucoma.

5. Patients with any other ocular pathology.

6. Patient using any artificial tear therapy.
Both groups were similar regarding age and gender characteristics. Informed consent in written and verbal was taken from the patients. University and Institute Ethics Committee approval was achieved.

First of all, Demographic data including age, gender, brief medical history including the use of topical drugs and duration were obtained from patient's medical records. Comprehensive ocular examination, Visual acuity in $\log$ mar, Intraocular pressure (IOP- Goldman applanation tonometry), tear breakup time (TBUT), Schirmer's II test (with anesthesia), Central corneal thickness (CCT) using Oculus pentacam, Endothelial cell count using Konan noncon Robo specular microscope, Corneal staining were done for both the groups.

\section{Results}

Table 1: Number of eyes on topical antiglaucoma therapy

\begin{tabular}{lc}
\hline Drugs & Number of Eyes \\
Timolol & 20 \\
Timolol \&Latanoprost & 12 \\
Timolol \&Brimonidine & 10 \\
Latanoprost\&Brimonidine & 8 \\
\hline
\end{tabular}

1. In (group I) 25 patients mean age of the patients was $44.16 \pm 7.22$. In (group II) 25 patients mean age of the patients was $44.16 \pm 7.22$.

2. In (group I) 25 patients (50 eyes) (56\%) were females and 25 patients (50 eyes) $(44 \%)$ were males. In (group II) 25 patients (50 eyes) $(56 \%)$ were females and 25 patients (50 eyes) $(44 \%)$ were males.

3. In the group, I (antiglaucoma eyes) 2.84 years was the mean duration of using drugs in these 25 patients (i.e. 1 year to 6years). Out of which 7 patients (14eyes) (28\%) were under treatment from 1 year, 4 patients ( 8 eyes) (16\%) were from 2 years, 6 patients (12 eyes) (24\%) were from 3 years and 4 patients ( 8 eyes) (16\%) were from 4 years, 2 patients (4 eyes) $(8 \%)$ were from 5 years and 2 patients ( 4 eyes) $(8 \%)$ were from 6 years.

4. In group $I-25$ patients (50 eyes) (antiglaucoma eyes) Patients diagnosed with primary open-angle glaucoma were 9 patients (18 eyes) is (36\%), patients diagnosed with primary angle-closure glaucoma were 5 patients (10 eyes) is (20\%)and patients diagnosed with ocular hypertension were 11 patients (22 eyes) is $44 \%$.

5. In group I (antiglaucoma group) Schirmer's II test showed 8 eyes (16\%) with zero dryness, 32 eyes $(64 \%)$ with mild to moderate dryness and 10 eyes (20\%) with severe dryness level were obtained.

6. In group I (antiglaucoma group) TBUT test showed 8 eyes $(16 \%)$ with zero level dryness, 32 eyes $(64 \%)$ with mild to moderate level dryness and 10 eyes (20\%) with severe level dryness were obtained. 
Table 2: Mean \pm SD of different test in group I and group II

\begin{tabular}{lccc} 
Tests & Antiglaucoma eyes group & Control eyes group & P \\
IOP $(\mathrm{mm} / \mathrm{hg})$ & $22.06 \pm 3.8778$ & $18.68 \pm 3.3285$ & 0.001 \\
Central Corneal Thickness $(\mu \mathrm{m})$ & $488.22 \pm 94.8006$ & $554.86 \pm 36.2919$ & 0.002 \\
Specular [average CD $\left(\right.$ cell//mm $\left.{ }^{2}\right)$ & $2273.36 \pm 165.2765$ & $2529.6 \pm 134.161$ & 0.001 \\
Schirmer's II test $(\mathrm{mm} / 5 \mathrm{~min})$ & $7.26 \pm 3.1996$ & $11.8 \pm 2.3647$ & 0.001 \\
TBUT $(\mathrm{sec})$ & $7.26 \pm 3.1932$ & $11.8 \pm 2.364$ & 0.001 \\
\hline
\end{tabular}

7. In group II (control group) Schirmer's II test showed 38 eyes $(76 \%)$ with zero dryness, 12 eyes $(24 \%)$ with mild to moderate dryness and 0 eyes $(0 \%)$ with severe dryness level were obtained.

8. In group II (control group) TBUT test showed 38 eyes $(76 \%)$ with zero level dryness, 12 eyes $(24 \%)$ with mild to moderate level dryness and 0 eyes $(0 \%)$ with severe level dryness were obtained.

9. Mann-Whitney U test value for IOP is 704; $(\mathrm{p}=0.001)$, CCT is 803; $(\mathrm{p}=0.002)$, Endothelial cell count is $274 ;(\mathrm{p}=0.001)$, Schirmer's II (with anesthesia) is 339.5; $(\mathrm{p}=0.001)$ and TBUT is 339; $(\mathrm{p}=0.001)$, respectively and all this were statistically significant.

10. Corneal staining score (NEI scale) in group I is 28 eyes as grade 0 (no stain), 17 eyes as grade 1(mild stain), 5 eyes as grade 2 (moderate stain) and zero eyes as severe stain were obtained. Corneal staining score (NEI scale) in group II is 47 eyes as grade 0 (no stain), 3 eyes as grade 1 (mild stain), zero eyes as grade 2 (moderate stain) and zero eyes as severe stain were obtained.

11. Fisher's Exact Test value is $19874(\mathrm{p}=0001$ is statistically significant.

12. CCT was compared to the different Diagnosis [mean $\pm \mathrm{SD}$ for Ocular hypertension(diagnosis 1) is 582.773 \pm 38.2186 , mean \pm SD for primary open-angle glaucoma (diagnosis 2) is $425.611 \pm 30.5828$ and mean \pm SD for primary angle-closure glaucoma (diagnosis 3) is $392.9 \pm 62.977$ ), respectively. The Chi-Square test value is $36.915(\mathrm{p}=0.001)$. CCT was more in Ocular hypertension as compared to the other diagnosis.

13. Endothelial cell count was compared to the different Diagnosis [mean $\pm \mathrm{SD}$ for Ocular hypertension(diagnosis 1) is $2358.727 \pm 120.0417$, mean \pm SD for primary open-angle glaucoma (diagnosis 2 ) is $2163.444 \pm 181.0392$ and mean \pm SD for primary angle-closure glaucoma (diagnosis3) is 2283.4 $\pm 105.362]$, respectively. The Chi-Square value is $11.876(\mathrm{p}=0.003)$. Endothelial cell count was more in Ocular hypertension as compared to the other diagnosis.

\section{Discussion}

1. Kurna et al. In 2014 conducted a study in which they stated that using topical medications for the long term may adversely affect the ocular surface in chronic ophthalmic conditions, such as glaucoma. ${ }^{8,9}$

2. No statistically significant difference in mean $\mathrm{SCH}$ I and None of the patients had TBUT values less than $10 \mathrm{~mm}$ or Schirmer value less than $5 \mathrm{~mm}$ indicating dry eye.

3. In present study we found in group I (antiglaucoma group) Schirmer's II test showed 8 eyes (16\%) with zero dryness, 32 eyes $(64 \%)$ with mild to moderate dryness and 10 eyes (20\%) with severe dryness level were obtained.

4. In group II (control group) Schirmer's II test showed 38 eyes $(76 \%)$ with zero dryness, 12 eyes $(24 \%)$ with mild to moderate dryness and 0 eyes $(0 \%)$ with severe dryness level were obtained.

5. In group I (antiglaucoma group) TBUT test showed 8 eyes $(16 \%)$ with zero level dryness, 32 eyes $(64 \%)$ with mild to moderate level dryness and 10 eyes (20\%) with severe level dryness were obtained.

6. In group II (control group) TBUT test showed 38 eyes $(76 \%)$ with zero level dryness, 12 eyes (24\%) with mild to moderate level dryness and 0 eyes $(0 \%)$ with severe level dryness were obtained.

7. Kuppens et al. and Lee et al., the TBUT and Schirmer I tests didn't detect any alteration with beta-blocker eye drops. Kozobolis et al. showed that the patients receiving bimatoprost, travoprost and latanoprost, had a significantly reduced central corneal mechanical sensitivity, comparing with TBUT and Schirmer test. ${ }^{10}$

8. Stewart et al. showed that the cornea and nasal conjunctival staining from baseline to hour 0 and hour 1 on the healthy subjects was increased due to timolol maleate. ${ }^{11}$ Lee et al. in a study had found that corneal epithelial punctate erosion was seen more in eyes being instilled with any type of beta-blocker and with a shorter TBUT. ${ }^{12}$

9. In present study Corneal staining score (NEI scale) in group I is 28 eyes as grade 0 (no stain), 17 eyes as grade 1 (mild stain), 5 eyes as grade 2 (moderate stain) and zero eyes as severe stain were obtained. Corneal staining score (NEI scale) in group II is 47 eyes as grade 0 (no stain), 3 eyes as grade 1 (mild stain), zero eyes as grade 2 (moderate stain) and zero eyes as severe stain were obtained.

10. Fisher's Exact Test value is $19.874(\mathrm{p}=0.001)$ is statistically significant staining of cornea shows 
somewhat corneal defects (like epithelial) in the antiglaucoma group.

11. This study has some limitations. There was no control group of glaucoma patients and the number of patients for each group was limited.The use of eye drops with and without preservatives had no relationship between them and to further evaluate the effect of antiglaucoma drugs with and without preservatives on the cornea and tear film.

12. Longer duration and a large number of patients were required to prove the actual effect of the antiglaucoma drug on the cornea and tear film and it may be beneficial to improve treatment for the patients using antiglaucoma drugs especially for a longer duration.

\section{Source of Funding}

None.

\section{Conflict of Interest}

None.

\section{References}

1. Quigley HA. The number of people with glaucoma worldwide in 2010 and 2020. Br J Ophthalmol. 2006;90(3):262-7.

2. Vinutha BV, Himamshu NVV, Sundeep, Niveditha H, Patil P, Liji P, et al. Prevalence of ocular surface disease in glaucoma patients using anti-glaucoma medications. J Evol Med Dent Sci. 2013;2(24):430814.

3. Pisella PJ. Prevalence of ocular symptoms and signs with preserved and preservative free glaucoma medication. $\mathrm{Br} J$ Ophthalmol. 2002;86(4):418-23.

4. Janson BJ, Alward WL, Kwon YH, Bettis DI, Fingert JH, Provencher LM, et al. Glaucoma-associated corneal endothelial cell damage: A review. Surv Ophthalmol. 2018;63(4):500-6.
5. Pandey AN, Sujata S. Study of long term structural and functional changes in medically controlled glaucoma. Int J Ophthalmol. 2014;7(1):128-32.

6. Yee RW. The effect of drop vehicle on the efficacy and side effects of topical glaucoma therapy: A review. Curr Opin Ophthalmol . 2007;18(2):134-9.

7. Detry-Morel M. Side effects of glaucoma medications. Bull Soc Belge Ophtalmol. 2006;299:27-40.

8. Martone G, Frezzotti P, Tosi GM. An in vivo confocal microscopy analysis of the effects of topical antiglaucoma therapy with preservative on corneal innervation and morphology. Am J Ophthalmol. 2009;147(4):725-35.

9. Kurna SA, Acikgoz S, Altun A, Ozbay N, Sengor T, Olcaysu OO. The effects of topical antiglaucoma drugs as monotherapy on the ocular surface: A prospective study. J Ophthalmol. 2014;2014:460-83.

10. Kozobolis VP, Detorakis ET, Maskaleris G, Koukoula SC, Fountoulakis N, Chrysochoou F, et al. Corneal sensitivity changes following the instillation of latanoprost, bimatoprost, and travoprost eyedrops. Am J Ophthalmol. 2005;139(4):742-3.

11. Stewart WC, Stewart JA, Holmes KT, Leech JN. Differences in ocular surface irritation between timolol hemihydrate and timolol maleate. Am J Ophthalmol. 2000;130(6):712-6.

12. Lee S, Kim MK, Choi HJ, Wee WR, Dm K. Comparative crosssectional analysis of the effects of topical antiglaucoma drugs on the ocular surface. Adv Ther. 2013;30(4):420-9.

\section{Author biography}

Veshal Madan Professor

Shubhangi Katare Senior Research Fellow

Neeta Mishra Associate Professor

Cite this article: Madan V, Katare S, Mishra N. Assessing the effect of anti-glaucoma drug on corneal morphology and tear film function. Indian J Clin Exp Ophthalmol 2020;6(2):291-294. 